Supporting Information for Org. Lett. (o10485363)

\title{
Efficient Heterogeneous Oxidation of Alkylarenes with Molecular Oxygen
}

\author{
Keigo Kamata, ${ }^{\dagger}$ Jun Kasai, ${ }^{\dagger}$ Kazuya Yamaguchi, ${ }^{\dagger,}$ and Noritaka Mizuno ${ }^{*, \dagger}, \dot{\dagger}$
}

+Core Research for Evolutional Science and Technology, Japan Science and Technology Agency, 4-1-8 Honcho, Kawaguchi, Saitama, 332-0012, Japan

${ }^{*}$ Department of Applied Chemistry, School of Engineering, The University of Tokyo, 7-3-1 Hongo, Bunkyo-ku, Tokyo, 113-8656, Japan

\section{Experimental Section}

Preparation of catalyst: $\mathrm{Ru}(\mathrm{OH})_{\mathrm{X}} / \mathrm{Al}_{2} \mathrm{O}_{3}$ catalyst was prepared according to the procedure reported previously. ${ }^{14} 2.0 \mathrm{~g}$ of $\gamma-\mathrm{Al}_{2} \mathrm{O}_{3}\left(\mathrm{JRC}-\mathrm{ALO}-4\right.$, BET surface area: $\left.174 \mathrm{~m}^{2} \cdot \mathrm{g}^{-1}\right)$ was vigorously stirred with $60 \mathrm{~mL}$ of an aqueous solution of $\mathrm{RuCl}_{3}\left(8.3 \times 10^{-3} \mathrm{M}\right)$ at room temperature. After $15 \mathrm{~min}$, the $\mathrm{pH}$ of the solution was slowly adjusted to 13.2 by addition of an aqueous solution of $\mathrm{NaOH}(1.0 \mathrm{M})$ and the resulting slurry was stirred for additional $24 \mathrm{~h}$.

The solid was then filtered off, washed with a large amount of water, and dried in vacuo to afford $2.1 \mathrm{~g}$ of $\mathrm{Ru}(\mathrm{OH})_{\mathrm{x}} / \mathrm{Al}_{2} \mathrm{O}_{3}$ as a dark green powder. The contents of ruthenium was 2.0 $w t \%$. The BET surface area was $182 \mathrm{~m}^{2} \cdot \mathrm{g}^{-1}$. The XRD pattern of $\mathrm{Ru}(\mathrm{OH})_{\mathrm{x}} / \mathrm{Al}_{2} \mathrm{O}_{3}$ was the same as that of the $\gamma-\mathrm{Al}_{2} \mathrm{O}_{3}$ support and no signal due to $\mathrm{Ru}$ metal (clusters) and $\mathrm{RuO}_{2}$ was observed. Particles of $\mathrm{Ru}$ metal (clusters) and $\mathrm{RuO}_{2}$ were not detected by TEM micrograph. 
X-ray photoelectron spectroscopy (XPS) shows that a peak of $3 \mathrm{~d}_{5 / 2}$ of $\mathrm{Ru}(0)$ is distinctly detected at $280.0 \mathrm{eV} .{ }^{15} \mathrm{Ru}(\mathrm{OH})_{\mathrm{X}} / \mathrm{Al}_{2} \mathrm{O}_{3}$ showed the peak at $281.9 \mathrm{eV}$, showing that the oxidation state of ruthenium species on $\mathrm{Ru}(\mathrm{OH})_{\mathrm{x}} / \mathrm{Al}_{2} \mathrm{O}_{3}$ is not zero. The ESR spectrum showed a broad signal at $\mathrm{g}=2.11$, which is assigned to $\mathrm{Ru}^{3+}$ with low-spin $\mathrm{d}^{5}$ electron configuration. $^{16}$ The IR spectrum showed a very broad $v(\mathrm{OH})$ band in the range of $3000-3700 \mathrm{~cm}^{-1}$. These facts suggest that ruthenium (III) hydroxide is highly dispersed on $\gamma-\mathrm{Al}_{2} \mathrm{O}_{3}$

Other transition metal $(\mathrm{Pd}, \mathrm{Rh}, \mathrm{Pt}, \mathrm{V}, \mathrm{Fe}$, and $\mathrm{Cu})$ hydroxide supported on alumina catalysts were prepared via similar procedure for $\mathrm{Ru}(\mathrm{OH})_{\mathrm{x}} / \mathrm{Al}_{2} \mathrm{O}_{3}$.

Preparation of amine-stabilized zero valent Ru clusters: Amine-stabilized zero valent Ru clusters were prepared according to the literature procedure. ${ }^{17}$ A solution of tetraoctylammonium bromide $(0.72 \mathrm{mmol})$ in toluene $(20 \mathrm{~mL})$ was added to a stirred solution of $\mathrm{RuCl}_{3}(0.48 \mathrm{mmol})$ in water $(20 \mathrm{~mL})$. After stirring the mixture for $12 \mathrm{~h}$ at room temperature, a solution of dodecylamine $(1.44 \mathrm{mmol})$ in toluene $(20 \mathrm{~mL})$ was added, and the mixture was vigorously stirred for an additional $1 \mathrm{~h}$. A solution of $\mathrm{NaBH}_{4}(4.3 \mathrm{mmol})$ in water $(20 \mathrm{~mL})$ was added to the above solution and then stirred at $373 \mathrm{~K}$. After $2 \mathrm{~h}$, solvent was evaporated under reduced pressure to ca. $5 \mathrm{~mL}$, and the residue was diluted with $30 \mathrm{~mL}$ of ethanol. The Ru clusters were separated by centrifugation and dried in a vacuum (Yield: 
$50 \mathrm{mg}$ ). The prepared $\mathrm{Ru}$ clusters was used as a catalyst for the aerobic oxidation of xanthene. It was found that the oxidation hardly proceeded under the conditions in Table 2.

Oxidation procedure: A typical procedure for the oxidation of alkylarenes was as follows: Into a glass vial were successively placed $\mathrm{Ru}(\mathrm{OH})_{\mathrm{x}} / \mathrm{Al}_{2} \mathrm{O}_{3}(\mathrm{Ru}: 2 \mathrm{~mol} \%)$, xanthene $(1 \mathrm{mmol})$, and $\mathrm{PhCF}_{3}(6 \mathrm{~mL})$. The reaction mixture was stirred at $373 \mathrm{~K}$ under 1 atm of molecular oxygen. The conversion and product selectivity were periodically determined by GC analysis during the oxidation. Consumption of molecular oxygen and formation of water (by TCD-GC) were also monitored during the oxidation. After the reaction was finished, the spent catalyst was separated by filtration, washed with a large amount of acetone, and dried in vacuo prior to being recycled. Then, the filtrate was evaporated in vacuo, and the crude product was purified by recrystallization to give analytically pure white crystals of xanthen-9-one ( $92 \%$ isolated yield).

All products are known and available. The products were confirmed by the comparison of their GC retention time, mass, ${ }^{1} \mathrm{H}$ and ${ }^{13} \mathrm{C}$ NMR spectroscopy with those of authentic samples. Purities of isolated products were determined by ${ }^{1} \mathrm{H}$ NMR and were $>95 \%$ in each case.

Xanthone: White powder. ${ }^{1} \mathrm{H}$ NMR $\left(270 \mathrm{MHz},\left[\mathrm{D}_{6}\right]\right.$ acetone, $25{ }^{\circ} \mathrm{C}$, TMS): $\delta$ 7.45-7.50 (m, $2 \mathrm{H}$, aromatic hydrogens), 7.59-7.62 (m, $2 \mathrm{H}$, aromatic hydrogens), 7.83-7.90 (m, $2 \mathrm{H}$, aromatic 
hydrogens), 8.25-8.28 (m, 2H, aromatic hydrogens); ${ }^{13} \mathrm{C}\left\{{ }^{1} \mathrm{H}\right\}$ NMR (67.5 MHz, [D 6 ]acetone, $\left.25^{\circ} \mathrm{C}, \mathrm{TMS}\right): \delta 117.9,121.7,123.8,126.6,134.7,156.1$ (aromatic carbons), 177.0 (carbonyl); MS (70 eV, EI): m/z (\%): 197 (15), $196(100)\left[M^{+}\right], 168$ (42), 139 (36).

Fluoren-9-one: Yellow powder. ${ }^{1} \mathrm{H}$ NMR $\left(270 \mathrm{MHz},\left[\mathrm{D}_{6}\right]\right.$ acetone, $\left.25{ }^{\circ} \mathrm{C}, \mathrm{TMS}\right): \delta$ 7.33-7.39 (m, 1H, aromatic hydrogen), 7.53-7.62 (m, 2H, aromatic hydrogens), 7.68-7.71 (m, $1 \mathrm{H}$, aromatic hydrogen); ${ }^{13} \mathrm{C}\left\{{ }^{1} \mathrm{H}\right\} \mathrm{NMR}\left(67.5 \mathrm{MHz},\left[\mathrm{D}_{6}\right]\right.$ acetone, $\left.25{ }^{\circ} \mathrm{C}, \mathrm{TMS}\right): \delta 121.6$, 124.6, 130.1, 134.7, 135.8, 145.2 (aromatic carbons), 193.8 (carbonyl); MS (70 eV, EI): m/z (\%): $181(14), 180(100)\left[M^{+}\right], 152(41), 151(21), 150$ (12), 76 (16).

Dibenzosuberone: Pale yellow liquid. ${ }^{1} \mathrm{H}$ NMR $\left(270 \mathrm{MHz},\left[\mathrm{D}_{6}\right]\right.$ acetone, $\left.25{ }^{\circ} \mathrm{C}, \mathrm{TMS}\right): \delta$ $3.12(\mathrm{~s}, 4 \mathrm{H}$, benzylic hydrogens), 7.22-7.46 (m, 6H, aromatic hydrogens), 7.93-7.96 (m, 2H, aromatic hydrogens); ${ }^{13} \mathrm{C}\left\{{ }^{1} \mathrm{H}\right\} \mathrm{NMR}\left(67.5 \mathrm{MHz},\left[\mathrm{D}_{6}\right]\right.$ acetone, $25{ }^{\circ} \mathrm{C}$, TMS): $\delta 35.3$ (benzylic carbons), 127.2, 130.2, 130.9, 133.1, 139.3, 142.8 (aromatic carbons), 195.3 (carbonyl); MS (70 eV, EI): m/z (\%): 209 (16), 208 (100) $\left[M^{+}\right], 207$ (34), 180 (57), 179 (59), 178 (45), 165 (31), 152 (15), 89 (23), 76 (11).

Anthracene: Pale yellow powder. ${ }^{1} \mathrm{H}$ NMR $\left(270 \mathrm{MHz},\left[\mathrm{D}_{6}\right]\right.$ acetone, $\left.25{ }^{\circ} \mathrm{C}, \mathrm{TMS}\right): \delta$ 7.49-7.52 (m, 4H, aromatic hydrogens), 8.06-8.09 (m, 2H, aromatic hydrogens); ${ }^{13} \mathrm{C}\left\{{ }^{1} \mathrm{H}\right\}$ NMR (67.5 MHz, [D $\left.\mathrm{D}_{6}\right]$ acetone, $\left.25^{\circ} \mathrm{C}, \mathrm{TMS}\right): \delta 126.3,127.0,129.0,132.7$ (aromatic carbons); MS (70 eV, EI): m/z (\%): 179 (16), $178(100)\left[M^{+}\right], 176(17), 89$ (11). 
9-Methylanthracene: Yellow powder. ${ }^{1} \mathrm{H}$ NMR (270 MHz, $\left[\mathrm{D}_{6}\right]$ acetone, $\left.25{ }^{\circ} \mathrm{C}, \mathrm{TMS}\right): \delta$ 3.05 (s, 3H, methyl hydrogens), 7.44-7.54 (m, 4H, aromatic hydrogens), 8.00-8.03 (m, 2H, aromatic hydrogens), 8.26-8.32 (m, 2H, aromatic hydrogens), 8.38 (brs, 1H, aromatic hydrogen); ${ }^{13} \mathrm{C}\left\{{ }^{1} \mathrm{H}\right\}$ NMR (67.5 MHz, [D 6 acetone, $25{ }^{\circ} \mathrm{C}$, TMS): $\delta 13.9$ (methyl carbon), 125.4, 125.7, 126.1, 126.2, 129.8, 131.0, 132.4 (aromatic carbons); MS (70 eV, EI): m/z (\%): $193(16), 192(100)\left[M^{+}\right], 191(58), 190(11), 189(25)$.

9-Phenylanthracene: Yellow powder. ${ }^{1} \mathrm{H}$ NMR (270 MHz, $\left[\mathrm{D}_{6}\right]$ acetone, $\left.25{ }^{\circ} \mathrm{C}, \mathrm{TMS}\right): \delta$ 7.37-7.67 (m, 11H, aromatic hydrogens), 8.11-8.15 (m, 2H, aromatic hydrogens), 8.62 (brs, $1 \mathrm{H}$, aromatic hydrogen); ${ }^{13} \mathrm{C}\left\{{ }^{1} \mathrm{H}\right\} \mathrm{NMR}\left(67.5 \mathrm{MHz},\left[\mathrm{D}_{6}\right]\right.$ acetone, $\left.25{ }^{\circ} \mathrm{C}, \mathrm{TMS}\right): \delta 126.1$, $126.4,127.2,127.5,128.4,129.3,129.4,131.0,131.9,132.4,137.7,139.6$ (aromatic carbons); MS (70 eV, EI): m/z (\%): 255 (22), 254 (100) $\left[M^{+}\right], 253(57), 252(41), 126(20)$.

Phenanthrene: White powder. ${ }^{1} \mathrm{H}$ NMR (270 MHz, [D 6 acetone, $25{ }^{\circ} \mathrm{C}$, TMS $): \delta 7.59-7.70$ (m, 4H, aromatic hydrogens), $7.80(\mathrm{~s}, 2 \mathrm{H}$, aromatic hydrogens), 7.93-7.96 (m, 2H, aromatic hydrogens), 8.76-8.79 (m, 2H, aromatic hydrogens); ${ }^{13} \mathrm{C}\left\{{ }^{1} \mathrm{H}\right\} \mathrm{NMR}\left(67.5 \mathrm{MHz},\left[\mathrm{D}_{6}\right]\right.$ acetone, $\left.25{ }^{\circ} \mathrm{C}, \mathrm{TMS}\right): \delta 123.5,127.5,127.6,127.7,129.4,131.1,133.0$ (aromatic carbons); MS (70

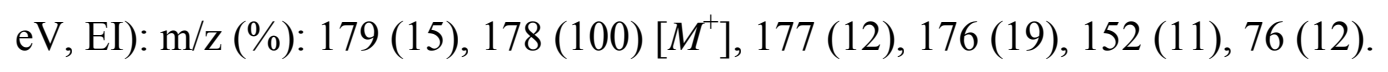

Naptharene: White powder. ${ }^{1} \mathrm{H}$ NMR $\left(270 \mathrm{MHz},\left[\mathrm{D}_{6}\right]\right.$ acetone, $25{ }^{\circ} \mathrm{C}$, TMS): $\delta 7.84-7.88$ (m, 4H, aromatic hydrogens), 7.44-7.49 (m, 4H, aromatic hydrogens); ${ }^{13} \mathrm{C}\left\{{ }^{1} \mathrm{H}\right\}$ NMR (67.5 
$\mathrm{MHz},\left[\mathrm{D}_{6}\right.$ ]acetone, $\left.25^{\circ} \mathrm{C}, \mathrm{TMS}\right): \delta 126.6,128.6,134.5$ (aromatic carbons); MS (70 eV, EI): $\mathrm{m} / \mathrm{z}(\%): 128(100)\left[M^{+}\right], 127(12)$

Quinoline: Colorless liquid. ${ }^{1} \mathrm{H}$ NMR (270 MHz, $\left[\mathrm{D}_{1}\right]$ chloroform, $\left.25{ }^{\circ} \mathrm{C}, \mathrm{TMS}\right): \delta$ 7.29-7.37 (m, 1H, aromatic hydrogen), 7.46-7.52 (m, 1H, aromatic hydrogen), 7.65-7.76 (m, $2 \mathrm{H}$, aromatic hydrogens), 8.06-8.13 (m, 2H, aromatic hydrogens), 8.88-8.90 (m, 1H, aromatic hydrogen); ${ }^{13} \mathrm{C}\left\{{ }^{1} \mathrm{H}\right\}$ NMR (67.5 MHz, [D 1 chloroform, $\left.25{ }^{\circ} \mathrm{C}, \mathrm{TMS}\right): \delta 120.8,126.3,127.6$, 128.0, 129.2, 135.8, 148.0, 150.2 (aromatic carbons); MS (70 eV, EI): m/z (\%): 129 (100) $\left[M^{+}\right], 128(19), 102(25)$.

Indole: White powder. ${ }^{1} \mathrm{H}$ NMR (270 MHz, [ $\left.\mathrm{D}_{1}\right]$ chloroform, $\left.25^{\circ} \mathrm{C}, \mathrm{TMS}\right): \delta 6.50-6.52(\mathrm{~m}$, $1 \mathrm{H}$, aromatic hydrogen), 7.01-7.03 (m, 1H, aromatic hydrogen), 7.07-7.27 (m, 3H, aromatic hydrogens), 7.61-7.65 (m, 1H, aromatic hydrogens), 7.76 (brs, $1 \mathrm{H}, \mathrm{NH}) ;{ }^{13} \mathrm{C}\left\{{ }^{1} \mathrm{H}\right\} \mathrm{NMR}(67.5$ MHz, $\left[\mathrm{D}_{1}\right]$ chloroform, $\left.25^{\circ} \mathrm{C}, \mathrm{TMS}\right): \delta 102.3,111.0,119.7,120.6,121.7,124.2,127.7,135.6$ (aromatic carbons); MS (70 eV, EI): m/z (\%): $117(100)\left[M^{+}\right], 90$ (41), 89 (27).

N-Benzylindole: Yellow oil. ${ }^{1} \mathrm{H}$ NMR $\left(270 \mathrm{MHz},\left[\mathrm{D}_{1}\right]\right.$ chloroform, $\left.25{ }^{\circ} \mathrm{C}, \mathrm{TMS}\right): \delta 4.18$ (s, $2 \mathrm{H}$, methylene hydrogens), 6.46-6.63 (3H, aromatic hydrogens), 6.95-7.06 (m, 3H, aromatic hydrogens), 7.20-7.33 (m, 5H, aromatic hydrogens); ${ }^{13} \mathrm{C}\left\{{ }^{1} \mathrm{H}\right\} \quad \mathrm{NMR} \quad(67.5 \mathrm{MHz}$, $\left[\mathrm{D}_{1}\right]$ chloroform, $\left.25^{\circ} \mathrm{C}, \mathrm{TMS}\right): \delta 53.6$ (methylene carbons), 102.2, 106.9, 117.5, 124.3, 124.4, 126.9, 127.1, 127.7, 128.3, 129.8, 138.3, 152.3 (aromatic carbons); MS (70 eV, EI): m/z (\%): 
208 (12), $207(64)\left[M^{+}\right], 91(100.00)$.

Larger scale reaction: Larger scale reaction (20-mmol scale) was performed with the same procedures as those above described. The turnover frequency (TOF) was determined from the initial rate $(\sim 1 \mathrm{~h})$. Reaction conditions for the larger scale reaction were as follows: Substrate $(20 \mathrm{mmol}), \mathrm{Ru}(\mathrm{OH})_{\mathrm{x}} / \mathrm{Al}_{2} \mathrm{O}_{3}(\mathrm{Ru}: 0.1 \% \mathrm{~mol}), o$-dichlorobenzene $(60 \mathrm{~mL}), 443 \mathrm{~K}$, under $1 \mathrm{~atm}$ of molecular oxygen.

Kinetic Studies: The reaction was periodically monitored by GC. Reaction conditions are given in the figure captions (Figures S3-S5). Reaction rates $\left(R_{0}\right)$ for the kinetic studies were determined from the slopes of reaction profiles ([substrate $]_{0}-[\text { substrate }]_{t} v s$. time plots) at low conversions $(<10 \%)$ of the substrate (initial rate method).

Kinetic Isotope Effect: A kinitic isotope effect $\left(k_{\mathrm{H}} / k_{\mathrm{D}}\right)$ was determined by the competitive oxidation of fluorene/fluorene- $d_{10}$. Reaction conditions were as follows: Fluorene/fluorene- $d_{10}(0.5 \mathrm{mmol} / 0.5 \mathrm{mmol}), \mathrm{Ru}(\mathrm{OH})_{\mathrm{x}} / \mathrm{Al}_{2} \mathrm{O}_{3}(2 \mathrm{~mol} \%), p$-xylene $(3 \mathrm{~mL}), 403$ $\mathrm{K}$, under 1 atm of molecular oxygen. The $k_{\mathrm{H}} / k_{\mathrm{D}}$ value was estimated from the slopes of reaction profiles $\left([\text { substrate }]_{0}-[\text { substrate }]_{t} v s\right.$. time plots) at low conversions $(<10 \%)$ of the both substrate (initial rate method). The error was estimated from the independent two runs. Synthesis of 9-hydroxy-9,10-dihydroanthracene: 9-Hydroxy-9,10-dihydroanthracene was synthesized by the reduction of anthraquinone using $\mathrm{Zn} / \mathrm{NH}_{3}$ by the reported procedure. ${ }^{18}$ Anthraquinone $(2.0 \mathrm{~g}, 9.6 \mathrm{mmol})$ and zinc metal ( $4 \mathrm{~g}$, activated with 5\% aqueous $\mathrm{HCl})$ were 
dissolved in $16 \%$ aqueous $\mathrm{NH}_{3}(20 \mathrm{~mL})$ at room temperature and then the resulting mixture was vigorously stirred under Ar at $343 \mathrm{~K}$. After $4 \mathrm{~h}$, unreacted zinc metal was filtered from the hot solution and washed with three $10 \mathrm{~mL}$ portions of hot dichloromethane. Then, the filtrate was extracted with dichloromethane $(20 \mathrm{~mL} \times 3)$. The extract was dried with calcium chloride and evaporated to give crude 9-hydroxy-9,10-dihydroanthracene as a yellow-white powder. The crude product was recrystallized from $n$-hexane/ether solution $(88 / 12 \mathrm{vol} / \mathrm{vol})$, giving $1.5 \mathrm{~g}$ of the purified compound as a white sticky crystal ( $80 \%$ yield). Detailed presentation of physical data of 9-hydroxy-9,10-dihydroanthracene: ${ }^{1} \mathrm{H}$ NMR $(270 \mathrm{MHz}$, $\left[\mathrm{D}_{1}\right]$ chloroform, $\left.25{ }^{\circ} \mathrm{C}, \mathrm{TMS}\right): \delta 2.03\left(\mathrm{~d},{ }^{3} \mathrm{~J}=6.75 \mathrm{~Hz}, 1 \mathrm{H}, \mathrm{OH}\right), 3.88-4.18(\mathrm{~m}, 2 \mathrm{H}$, benzylic hydrogens), $5.63\left(\mathrm{~d},{ }^{3} J=5.63 \mathrm{~Hz}, 1 \mathrm{H}\right.$, benzylic hydrogens), 7.28-7.39 (m, 6H, aromatic hydrogens), 7.60-7.67 (m, 2H, aromatic hydrogens); ${ }^{13} \mathrm{C}\left\{{ }^{1} \mathrm{H}\right\} \quad \mathrm{NMR} \quad(67.5 \mathrm{MHz}$, $\left[\mathrm{D}_{1}\right]$ chloroform, $\left.25{ }^{\circ} \mathrm{C}, \mathrm{TMS}\right): \delta 34.9$ (methylene carbon), 70.4 ( $\alpha$ carbon) $126.4,126.5,127.5$, 127.6, 135.7, 138.5 (aromatic carbons); elemental analysis calcd (\%) for $\mathrm{C}_{14} \mathrm{H}_{12} \mathrm{O}: \mathrm{C} 85.68, \mathrm{H}$ $6.16 \%$; found: C $85.66, \mathrm{H} 6.04 \%$.

Synthesis of 9-methyl-9,10-dihydroanthracene: 9-Methyl-9,10-dihydroanthracene was synthesized by the reduction of 9-methylanthracene using $\mathrm{Na} / \mathrm{EtOH}$ by the reported procedure. $^{19}$ To a three-necked flask with a reflux condenser were placed 9-methylanthracene $(3.9 \mathrm{~g}, 20 \mathrm{mmol})$ and $60 \mathrm{~mL}$ of carefully dried ethanol under Ar. The 
suspension was stirred and heated to $323 \mathrm{~K}$, and $0.5 \mathrm{~g}$ of sodium was added every 5 minutes for 55 minutes (sodium, total $6 \mathrm{~g}, 0.26 \mathrm{mmol}$ ). Then, the reaction mixture was refluxed for 15 minutes (bath temp. $363 \mathrm{~K}$ ). The reaction mixture was then cooled and carefully diluted with $70 \mathrm{~mL}$ of deionized water. The yellow powder (a mixture of 9-methylanthracene and 9-methyl-9,10-dihydroanthracene) was collected by the filtration, washed with $100 \mathrm{~mL}$ of water, and dried in vacuo. The dried yellow powder was again suspended in $60 \mathrm{~mL}$ of carefully dried ethanol under Ar. The suspension was stirred and heated to $323 \mathrm{~K}$, and $0.5 \mathrm{~g}$ of sodium was added every 5 minutes for 55 minutes (sodium, total $6 \mathrm{~g}, 0.26 \mathrm{mmol}$ ). After the addition of sodium, the reaction mixture was refluxed for 15 minutes (bath temp. $363 \mathrm{~K}$ ). The reaction mixture was then cooled and carefully diluted with $70 \mathrm{~mL}$ of deionized water. The yellow powder (9-methyl-9,10-dihydroanthracene) was collected by the filtration, washed with $100 \mathrm{~mL}$ of water, and dried in vacuo. The crude yellow powder was then recrystallized from ethanol, giving $2.9 \mathrm{~g}$ of the purified 9-methyl-9,10-dihydroanthracene as a yellow sticky crystal $(75 \%$ yield). Detailed presentation of physical data of 9-methyl-9,10-dihydroanthracene: ${ }^{1} \mathrm{H}$ NMR $\left(270 \mathrm{MHz},\left[\mathrm{D}_{6}\right]\right.$ acetone, $\left.25{ }^{\circ} \mathrm{C}, \mathrm{TMS}\right): \delta 1.36(\mathrm{~d}$, ${ }^{3} J=7.07 \mathrm{~Hz}, 3 \mathrm{H}$, methyl hydrogens), 3.85-4.13 (m, 3H, benzylic hydrogens), 7.12-7.33 (m, $8 \mathrm{H}$, aromatic hydrogens); ${ }^{13} \mathrm{C}\left\{{ }^{1} \mathrm{H}\right\}$ NMR $\left(67.5 \mathrm{MHz},\left[\mathrm{D}_{6}\right]\right.$ acetone, $\left.25{ }^{\circ} \mathrm{C}, \mathrm{TMS}\right): \delta 24.0$ (methyl carbon), 35.4, 41.8 (benzylic carbons) 126.6, 127.1, 127.6, 128.3, 136.6, 142.5 
(aromatic carbons); MS (70 eV, EI): m/z (\%): 194 (12.75) $\left[M^{+}\right], 180$ (14.96), 179 (100.00)

$\left[M^{+}-\mathrm{CH}_{3}\right], 178$ (37.93); elemental analysis calcd (\%) for $\mathrm{C}_{15} \mathrm{H}_{14}: \mathrm{C}$ 92.74, $\mathrm{H}$ 7.26\%; found: C 92.53, H 7.26\%.

Synthesis of 9-phenyl-9,10-dihydroanthracene: 9-Phenyl-9,10-dihydroanthracene was synthesized by the same procedure as that for the synthesis of 9-methyl-9,10-dihydroanthracene (92\% yield). Detailed presentation of physical data of 9-phenyl-9,10-dihydroanthracene: $\delta$ 3.89-4.06 (m, 2H, benzylic hydrogens), $5.33(\mathrm{~s}, 1 \mathrm{H}$, benzylic hydrogen), 7.07-7.39 (m, 13H, aromatic hydrogens); ${ }^{13} \mathrm{C}\left\{{ }^{1} \mathrm{H}\right\}$ NMR $(67.5 \mathrm{MHz}$, $\left[\mathrm{D}_{6}\right]$ acetone, $25{ }^{\circ} \mathrm{C}, \mathrm{TMS}$ ): $\delta 35.8,52.0$ (benzylic carbons) 126.7, 126.9, 127.0, 128.3, 128.4, 128.9, 129.0, 137.0, 140.3, 144.9 (aromatic carbons); MS (70 eV, EI): m/z (\%): 256 (47.55) $\left[M^{+}\right], 179$ (63.09) $\left[M^{+}-\mathrm{Ph}\right], 178$ (100.00); elemental analysis calcd (\%) for $\mathrm{C}_{20} \mathrm{H}_{16}: \mathrm{C}$ 93.71, $\mathrm{H}$ 6.29\%; found: C 93.61, H 6.29\%.

Synthesis of $N$-benzylindoline: $N$-Benzylindoline was synthesized by the $N$-benzylation of indoline using benzyl chloride by the reported procedure. ${ }^{20}$ Indoline $(6.0 \mathrm{~g}, 50 \mathrm{mmol})$ was added to sodium bicarbonate $(5.25 \mathrm{~g}, 62.5 \mathrm{mmol})$ in $10 \mathrm{~mL}$ water and the mixture was stirred at $368 \mathrm{~K}$. Benzyl chloride $(6.3 \mathrm{~g}, 50 \mathrm{mmol})$ was added dropwise for $1.5 \mathrm{~h}$, and stirring was continued for an additional $3.5 \mathrm{~h}$. After cooling the mixture, the layers were separated, and the aqueous layer was extracted with ether $(20 \mathrm{~mL} \times 3)$. The combined organic layers were 
dried over calcium chloride and evaporated to give $8.9 \mathrm{~g}$ of $\mathrm{N}$-benzylindoline as purple oil (85\% yield). Detailed presentation of physical data of $N$-benzylindoline: ${ }^{1} \mathrm{H}$ NMR (270 MHz, $\left[\mathrm{D}_{1}\right]$ chloroform, $\left.25{ }^{\circ} \mathrm{C}, \mathrm{TMS}\right): \delta 2.86\left(\mathrm{t},{ }^{3} \mathrm{~J}=8.23 \mathrm{~Hz}, 2 \mathrm{H}\right.$, methylene hydrogens $), 3.24$ (t, ${ }^{3} J=8.23 \mathrm{~Hz}, 2 \mathrm{H}$, methylene hydrogens), 4.18 (s, 2H, methylene hydrogens), 6.46 (d, ${ }^{3} J=$ $7.74 \mathrm{~Hz}, 1 \mathrm{H}$, aromatic hydrogen), $6.63\left(\mathrm{t},{ }^{3} \mathrm{~J}=7.24 \mathrm{~Hz}, 1 \mathrm{H}\right.$, aromatic hydrogen), 6.98-7.06 (m, $2 \mathrm{H}$, aromatic hydrogens), 7.20-7.33 (m, 5H, aromatic hydrogens); ${ }^{13} \mathrm{C}\left\{{ }^{1} \mathrm{H}\right\} \mathrm{NMR}(67.5 \mathrm{MHz}$, $\left[\mathrm{D}_{1}\right]$ chloroform, $\left.25{ }^{\circ} \mathrm{C}, \mathrm{TMS}\right): \delta 28.5,53.5,53.6$ (methylene carbons), 106.9, 117.5, 124.3, 126.9, 127.1, 127.7, 128.3, 129.8, 138.3, 152.3 (aromatic carbons); MS (70 eV, EI): m/z (\%): 210 (14.77), 209 (91.07) $\left[M^{+}\right], 208$ (20.34), 132 (47.94), 118 (42.46), 117 (17.33), 92 (14.77), $91(100.00), 65(16.44)$. 


\section{References}

(15) Wagner, C. D.; Riggs, W. M.; Davis, L. E.; Moulder, F. Handbook of X-ray Photoelectron Spectroscopy (Ed: G. E. Muilenberg), Perkin-Elmer Corporation, Physical Electronics Division, Eden Prairie, Minn. 1979.

(16) (a) Wan, B.-Z.; Lunsford, J. H. Inorg. Chim. Acta 1982, 65, 29. (b) Cattania, M. G.; Gervasini, A.; Morazzoni, F.; Scotti, P.; Strumolo, D. J. Chem. Soc., Faraday Trans. 1 1987, 83,3619 .

(17) Na, Y.; Park, S.; Han, S. B.; Han, H.; Ko, S.; Chang, S. J. Am. Chem. Soc. 2004, 126, 250.

(18) McCormack, A. C.; McDonnell, C. M.; O’Ferrall, R. A. M.; O’Donoghue, A. C.; Rao, S. N. J. Am. Chem. Soc. 2002, 124, 8575.

(19) Bass, K. C. Org. Synth., Vol. 5, Wiley, New York, 1973, p.398-400.

(20) Belsky, I.; Gertner, D.; Zilkha, A. J. Org. Chem. 1968, 33, 13487

(21) Cheng, J. P.; Handoo, K. L.; Parker, V. D. J. Am. Chem. Soc. 1993, 115, 2655.

(22) Handoo, K. L.; Cheng, J. P.; Parker, V. D. J. Am. Chem. Soc. 1993, 115, 5067.

(23) Alnajjar, M. S.; Zhang, X.-M.; Gleicher, G. J.; Truksa, S. V.; Franz, J. A. J. Org. Chem. 2002, 67, 9016

(24) Buncel, E.; Venkatachalam, T. K. J. Organomet. Chem. 2000, 604, 208. 


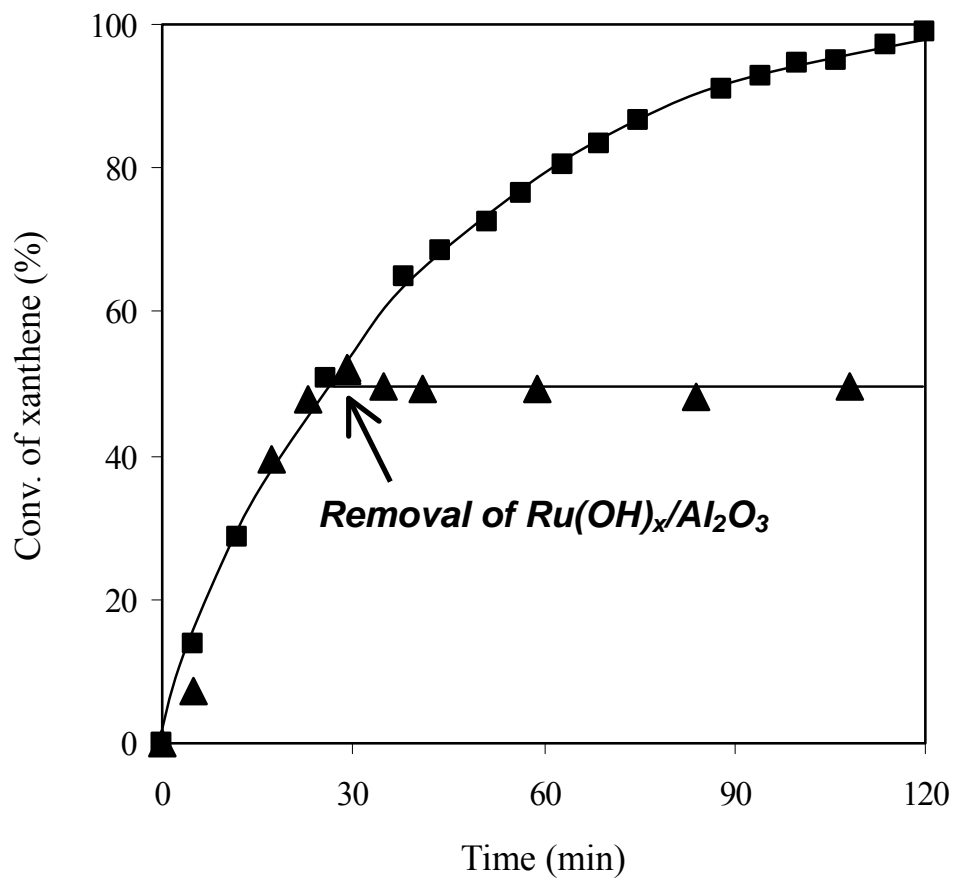

Figure S1. Effect of removal of $\mathrm{Ru}(\mathrm{OH})_{\mathrm{x}} / \mathrm{Al}_{2} \mathrm{O}_{3}$ on the oxidation of xanthene to xanthen-9-one. Without removal of $\mathrm{Ru}(\mathrm{OH})_{\mathrm{x}} / \mathrm{Al}_{2} \mathrm{O}_{3}(\mathbf{\square})$; an arrow indicates the removal of $\mathrm{Ru}(\mathrm{OH})_{\mathrm{x}} / \mathrm{Al}_{2} \mathrm{O}_{3}(\boldsymbol{\Delta})$. Reaction conditions were as follows: Xanthene (1 $\left.\mathrm{mmol}\right)$, $\mathrm{Ru}(\mathrm{OH})_{\mathrm{x}} / \mathrm{Al}_{2} \mathrm{O}_{3}(2 \mathrm{~mol} \%), \mathrm{PhCF}_{3}(6 \mathrm{~mL}), 373 \mathrm{~K}$, under $1 \mathrm{~atm}$ of molecular oxygen. 

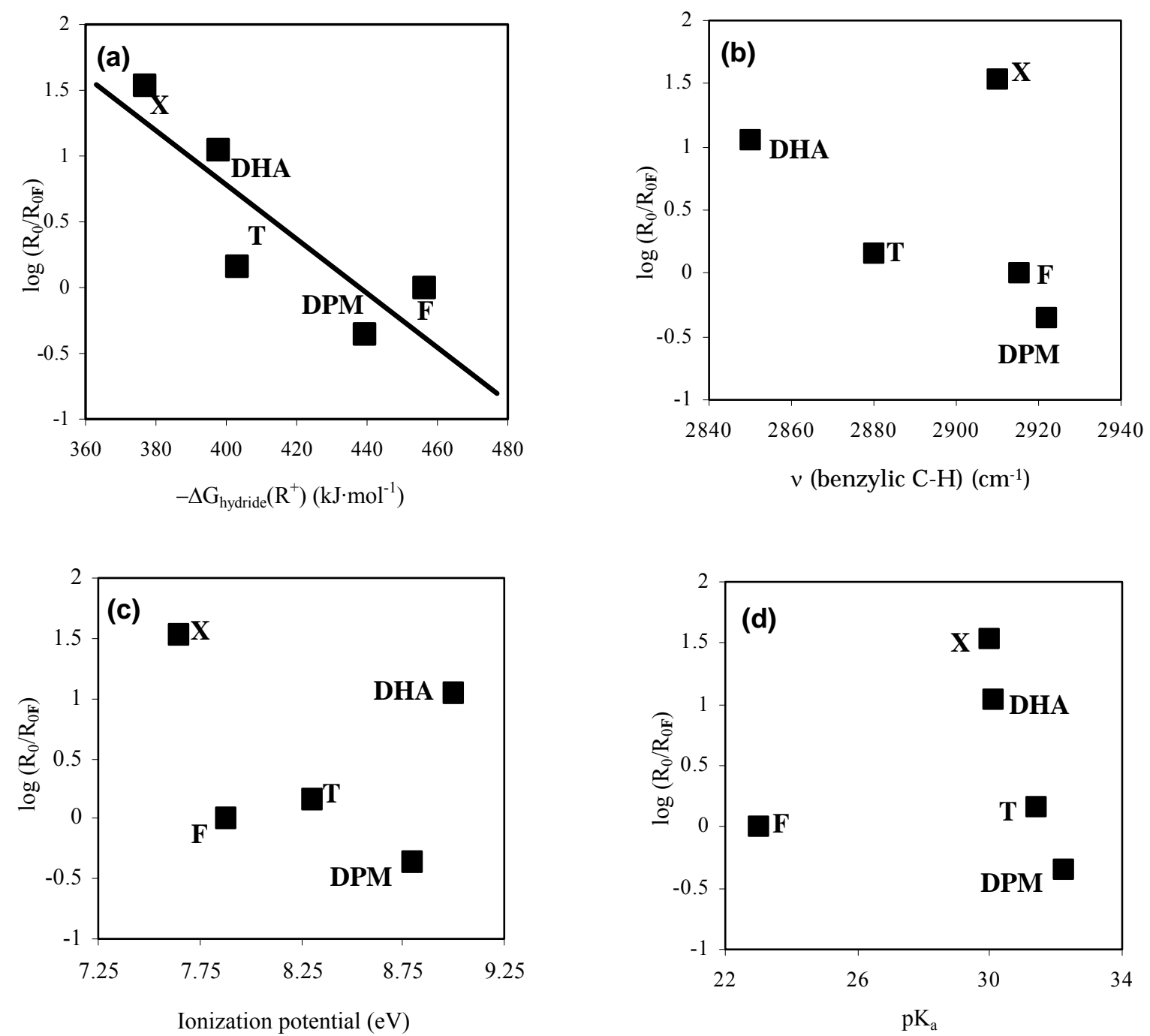

Figure S2. The relative rates for the competitive oxidations of alkylarenes, xanthene $(\mathbf{X})$, 9,10-dihydroanthracene (DHA), triphenylmethane (T), diphenylmethane (DPM), and fluorene (F), as a function of heterolytic (a) and homolytic bond energy (b), ionization potential (c), and $\mathrm{pK}_{\mathrm{a}}(\mathrm{d})$. Reaction conditions were as follows: Alkylarene/fluorene $(0.5 \mathrm{mmol} / 0.5 \mathrm{mmol}), \mathrm{Ru}(\mathrm{OH})_{\mathrm{x}} / \mathrm{Al}_{2} \mathrm{O}_{3}(2$ mol\%), $p$-xylene $(6 \mathrm{~mL}), 403 \mathrm{~K}$, under $1 \mathrm{~atm}$ of molecular oxygen. The initial rates $(-d[$ substrate $] / d t$, $R_{0}$ ) were determined from the slopes of reaction profiles ([substrate $]_{0}-[\text { substrate }]_{t} v s$. time plots) at low conversions $(<10 \%)$ of the substrates. $-\Delta \mathrm{G}_{\text {hydride }}\left(\mathrm{R}^{+}\right)$is the free energy of R-H heterolytic bond disassociation. Data of $-\Delta \mathrm{G}_{\text {hydride }}\left(\mathrm{R}^{+}\right)$are from references 21 and 22. Date of $v($ benzylic $\mathrm{C}-\mathrm{H})$ and ionization potential are from NIST at http://webbook.nist.gov/chemistry. Data of $\mathrm{pK}_{\mathrm{a}}$ are taken from references 23 and 24 . 


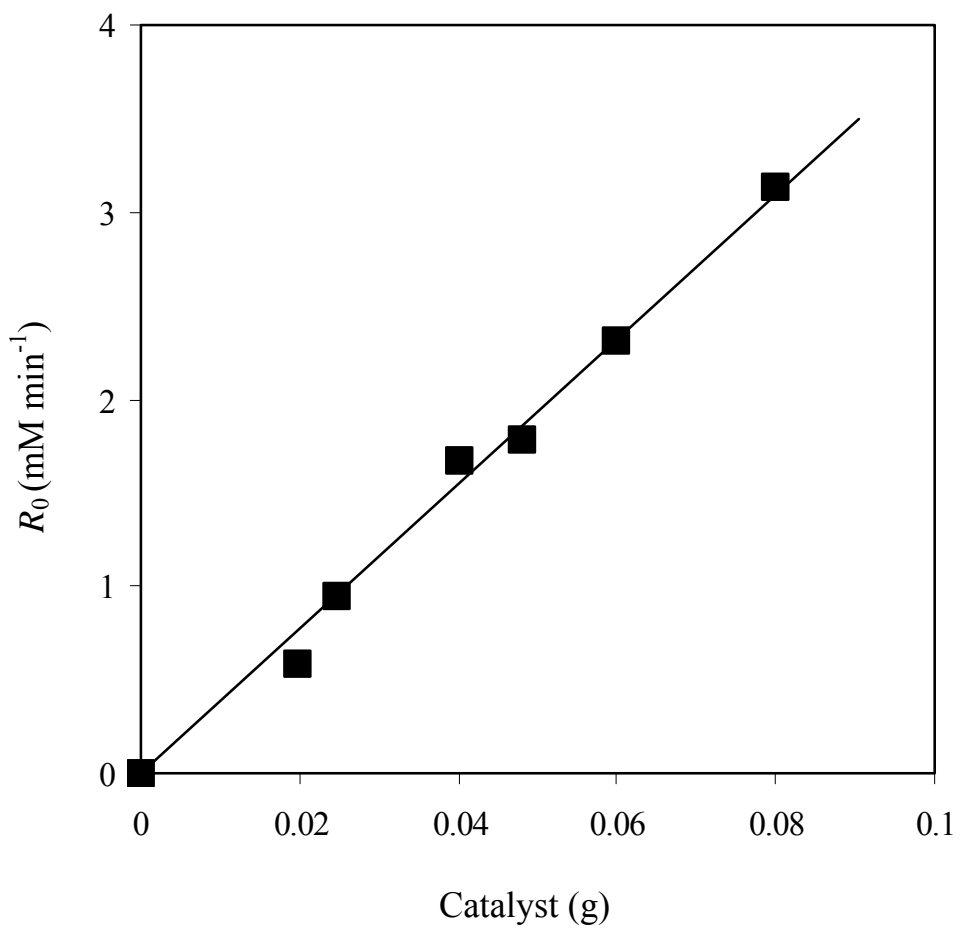

Figure S3. Dependence of reaction rate on amounts of catalyst Reaction conditions: Xanthene (0.17 M), $\mathrm{Ru}(\mathrm{OH})_{\mathrm{x}} / \mathrm{Al}_{2} \mathrm{O}_{3}(\sim 0.08 \mathrm{~g}, \sim 2 \mathrm{~mol} \%) \mathrm{PhCF}_{3}(6 \mathrm{~mL}), 373 \mathrm{~K}$, under $1 \mathrm{~atm}$ of molecular oxygen. 


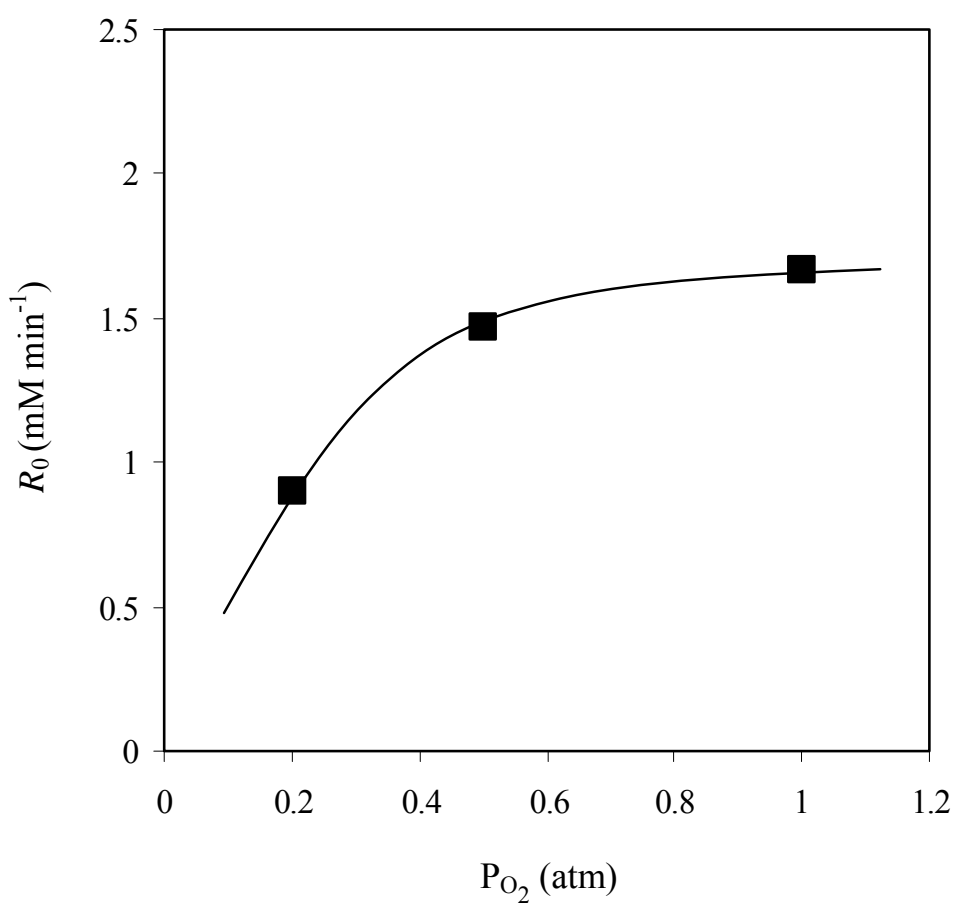

Figure S4. Dependence of reaction rate on $\mathrm{P}_{\mathrm{O}_{2}}$. Reaction conditions: Xanthene $(0.17 \mathrm{M})$, $\mathrm{Ru}(\mathrm{OH})_{\mathrm{x}} / \mathrm{Al}_{2} \mathrm{O}_{3}(2 \mathrm{~mol} \%), \mathrm{PhCF}_{3}(6 \mathrm{~mL}), 373 \mathrm{~K}, \mathrm{P}_{\mathrm{O}_{2}}(0.2-1.0 \mathrm{~atm})$. 


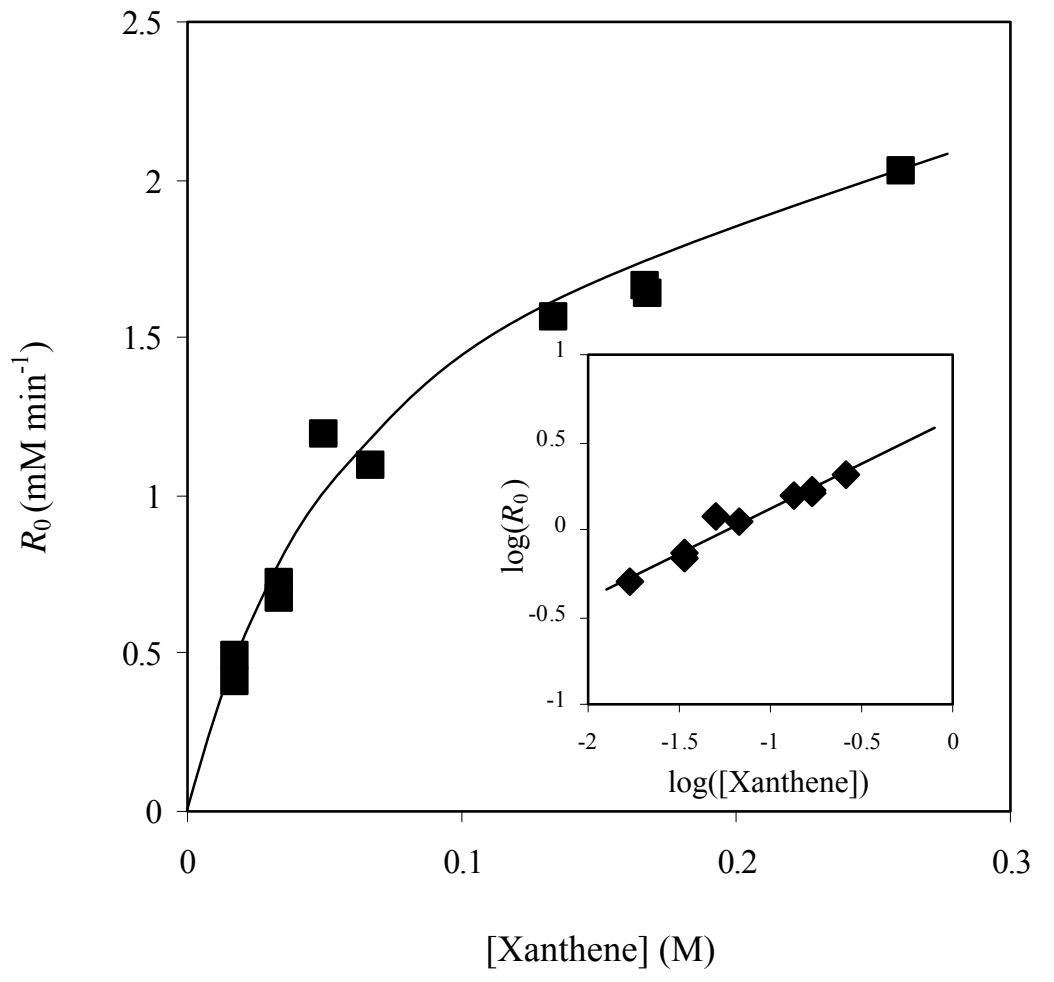

Figure S5. Dependence of reaction rate on concentration of xanthene. Reaction conditions: Xanthene (0.017-0.30 M), $\mathrm{Ru}(\mathrm{OH})_{\mathrm{x}} / \mathrm{Al}_{2} \mathrm{O}_{3}(2 \mathrm{~mol} \%) \mathrm{PhCF}_{3}(6 \mathrm{~mL}), 373 \mathrm{~K}$, under 1 atm of molecular oxygen. Inset, $\log \left(R_{0}\right) v s . \log ([\mathrm{X} a n t h e n e])$ plots. Slope from the inset $=0.51$. 


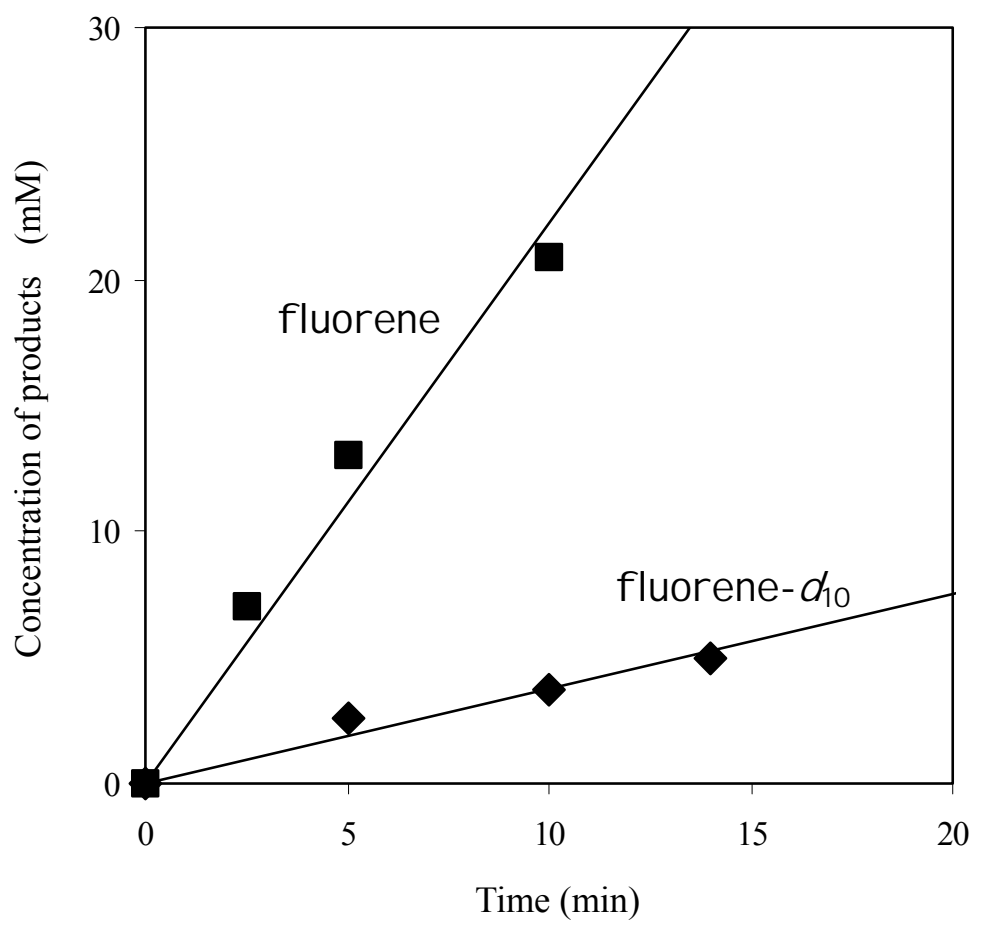

Figure S6. Competitive oxidation of fluorene and fluorene- $d_{10}$. Reaction conditions were as follows: Fluorene/fluorene- $d_{10}(0.5 \mathrm{mmol} / 0.5 \mathrm{mmol}), \mathrm{Ru}(\mathrm{OH})_{\mathrm{x}} / \mathrm{Al}_{2} \mathrm{O}_{3}(2 \mathrm{~mol} \%), p$-xylene $(3 \mathrm{~mL}), 403 \mathrm{~K}$, under $1 \mathrm{~atm}$ of molecular oxygen. 